\title{
Restriction by Noncontraction
}

\author{
Elia Zardini
}

\begin{abstract}
This paper investigates how naive theories of truth fare with respect to a set of extremely plausible principles of restricted quantification. It is first shown that both nonsubstructural theories as well as certain substructural theories cannot validate all those principles. Then, pursuing further an approach to the semantic paradoxes that the author has defended elsewhere, the theory of restricted quantification available in a specific naive theory that rejects the structural property of contraction is explored. It is shown that the theory validates all the principles in question, and it is argued that other prima facie plausible principles that the theory fails to validate are objectionable on independent grounds.
\end{abstract}

\section{Implication}

An operation of implication $^{1}$ has traditionally been associated with at least six different (possibly related) functions (which I will refer to as 'the Functions'). Taking an implicational proposition expressed by the conditional $\varphi \rightarrow \psi$, these can informally be expressed as:

SUFFICIENT CONDITION $\Rightarrow$ : Be at least as weak as $\varphi$ being a sufficient condition for $\psi$;

SUFFICIENT CONDITION ${ }^{\Leftarrow}$ : Be at least as strong as $\varphi$ being a sufficient condition for $\psi$;

NO REFUTATION $\Rightarrow$ : Follow from its not being the case that $\varphi$ holds while $\psi$ does not; ${ }^{2}$

NO REFUTATION ${ }^{\leftarrow}$ : Entail that it is not the case that $\varphi$ holds while $\psi$ does not;

DEDUCTION THEOREM: Be at least as weak as $\varphi$ entailing $\psi$;

MODUS PONENS: Together with $\varphi$, logically necessitate $\psi$. $^{3}$

Received September 1, 2012; accepted October 14, 2013

First published online January 28, 2016

2010 Mathematics Subject Classification: Primary 03; Secondary A05, B20, B47, B53, B65

Keywords: analetheism, contraction, dialetheism, naive truth, restricted quantification, substructural logics, transitivity

(C) 2016 by University of Notre Dame $\quad$ 10.1215/00294527-3429057 


\title{
Restriction by Noncontraction
}

\author{
Elia Zardini
}

\begin{abstract}
This paper investigates how naive theories of truth fare with respect to a set of extremely plausible principles of restricted quantification. It is first shown that both nonsubstructural theories as well as certain substructural theories cannot validate all those principles. Then, pursuing further an approach to the semantic paradoxes that the author has defended elsewhere, the theory of restricted quantification available in a specific naive theory that rejects the structural property of contraction is explored. It is shown that the theory validates all the principles in question, and it is argued that other prima facie plausible principles that the theory fails to validate are objectionable on independent grounds.
\end{abstract}

\section{Implication}

An operation of implication $^{1}$ has traditionally been associated with at least six different (possibly related) functions (which I will refer to as 'the Functions'). Taking an implicational proposition expressed by the conditional $\varphi \rightarrow \psi$, these can informally be expressed as:

SUFFICIENT CONDITION $\Rightarrow$ : Be at least as weak as $\varphi$ being a sufficient condition for $\psi$;

SUFFICIENT CONDITION ${ }^{\Leftarrow}$ : Be at least as strong as $\varphi$ being a sufficient condition for $\psi$;

NO REFUTATION $\Rightarrow$ : Follow from its not being the case that $\varphi$ holds while $\psi$ does not; ${ }^{2}$

NO REFUTATION ${ }^{\leftarrow}$ : Entail that it is not the case that $\varphi$ holds while $\psi$ does not;

DEDUCTION THEOREM: Be at least as weak as $\varphi$ entailing $\psi$;

MODUS PONENS: Together with $\varphi$, logically necessitate $\psi$. $^{3}$

Received September 1, 2012; accepted October 14, 2013

First published online January 28, 2016

2010 Mathematics Subject Classification: Primary 03; Secondary A05, B20, B47, B53, B65

Keywords: analetheism, contraction, dialetheism, naive truth, restricted quantification, substructural logics, transitivity

(C) 2016 by University of Notre Dame $\quad$ 10.1215/00294527-3429057 


\title{
Restriction by Noncontraction
}

\author{
Elia Zardini
}

\begin{abstract}
This paper investigates how naive theories of truth fare with respect to a set of extremely plausible principles of restricted quantification. It is first shown that both nonsubstructural theories as well as certain substructural theories cannot validate all those principles. Then, pursuing further an approach to the semantic paradoxes that the author has defended elsewhere, the theory of restricted quantification available in a specific naive theory that rejects the structural property of contraction is explored. It is shown that the theory validates all the principles in question, and it is argued that other prima facie plausible principles that the theory fails to validate are objectionable on independent grounds.
\end{abstract}

\section{Implication}

An operation of implication $^{1}$ has traditionally been associated with at least six different (possibly related) functions (which I will refer to as 'the Functions'). Taking an implicational proposition expressed by the conditional $\varphi \rightarrow \psi$, these can informally be expressed as:

SUFFICIENT CONDITION $\Rightarrow$ : Be at least as weak as $\varphi$ being a sufficient condition for $\psi$;

SUFFICIENT CONDITION ${ }^{\Leftarrow}$ : Be at least as strong as $\varphi$ being a sufficient condition for $\psi$;

NO REFUTATION $\Rightarrow$ : Follow from its not being the case that $\varphi$ holds while $\psi$ does not; ${ }^{2}$

NO REFUTATION ${ }^{\leftarrow}$ : Entail that it is not the case that $\varphi$ holds while $\psi$ does not;

DEDUCTION THEOREM: Be at least as weak as $\varphi$ entailing $\psi$;

MODUS PONENS: Together with $\varphi$, logically necessitate $\psi$. $^{3}$

Received September 1, 2012; accepted October 14, 2013

First published online January 28, 2016

2010 Mathematics Subject Classification: Primary 03; Secondary A05, B20, B47, B53, B65

Keywords: analetheism, contraction, dialetheism, naive truth, restricted quantification, substructural logics, transitivity

(C) 2016 by University of Notre Dame $\quad$ 10.1215/00294527-3429057 


\title{
Restriction by Noncontraction
}

\author{
Elia Zardini
}

\begin{abstract}
This paper investigates how naive theories of truth fare with respect to a set of extremely plausible principles of restricted quantification. It is first shown that both nonsubstructural theories as well as certain substructural theories cannot validate all those principles. Then, pursuing further an approach to the semantic paradoxes that the author has defended elsewhere, the theory of restricted quantification available in a specific naive theory that rejects the structural property of contraction is explored. It is shown that the theory validates all the principles in question, and it is argued that other prima facie plausible principles that the theory fails to validate are objectionable on independent grounds.
\end{abstract}

\section{Implication}

An operation of implication $^{1}$ has traditionally been associated with at least six different (possibly related) functions (which I will refer to as 'the Functions'). Taking an implicational proposition expressed by the conditional $\varphi \rightarrow \psi$, these can informally be expressed as:

SUFFICIENT CONDITION $\Rightarrow$ : Be at least as weak as $\varphi$ being a sufficient condition for $\psi$;

SUFFICIENT CONDITION ${ }^{\Leftarrow}$ : Be at least as strong as $\varphi$ being a sufficient condition for $\psi$;

NO REFUTATION $\Rightarrow$ : Follow from its not being the case that $\varphi$ holds while $\psi$ does not; ${ }^{2}$

NO REFUTATION ${ }^{\leftarrow}$ : Entail that it is not the case that $\varphi$ holds while $\psi$ does not;

DEDUCTION THEOREM: Be at least as weak as $\varphi$ entailing $\psi$;

MODUS PONENS: Together with $\varphi$, logically necessitate $\psi$. $^{3}$

Received September 1, 2012; accepted October 14, 2013

First published online January 28, 2016

2010 Mathematics Subject Classification: Primary 03; Secondary A05, B20, B47, B53, B65

Keywords: analetheism, contraction, dialetheism, naive truth, restricted quantification, substructural logics, transitivity

(C) 2016 by University of Notre Dame $\quad$ 10.1215/00294527-3429057 


\title{
Restriction by Noncontraction
}

\author{
Elia Zardini
}

\begin{abstract}
This paper investigates how naive theories of truth fare with respect to a set of extremely plausible principles of restricted quantification. It is first shown that both nonsubstructural theories as well as certain substructural theories cannot validate all those principles. Then, pursuing further an approach to the semantic paradoxes that the author has defended elsewhere, the theory of restricted quantification available in a specific naive theory that rejects the structural property of contraction is explored. It is shown that the theory validates all the principles in question, and it is argued that other prima facie plausible principles that the theory fails to validate are objectionable on independent grounds.
\end{abstract}

\section{Implication}

An operation of implication $^{1}$ has traditionally been associated with at least six different (possibly related) functions (which I will refer to as 'the Functions'). Taking an implicational proposition expressed by the conditional $\varphi \rightarrow \psi$, these can informally be expressed as:

SUFFICIENT CONDITION $\Rightarrow$ : Be at least as weak as $\varphi$ being a sufficient condition for $\psi$;

SUFFICIENT CONDITION ${ }^{\Leftarrow}$ : Be at least as strong as $\varphi$ being a sufficient condition for $\psi$;

NO REFUTATION $\Rightarrow$ : Follow from its not being the case that $\varphi$ holds while $\psi$ does not; ${ }^{2}$

NO REFUTATION ${ }^{\leftarrow}$ : Entail that it is not the case that $\varphi$ holds while $\psi$ does not;

DEDUCTION THEOREM: Be at least as weak as $\varphi$ entailing $\psi$;

MODUS PONENS: Together with $\varphi$, logically necessitate $\psi$. $^{3}$

Received September 1, 2012; accepted October 14, 2013

First published online January 28, 2016

2010 Mathematics Subject Classification: Primary 03; Secondary A05, B20, B47, B53, B65

Keywords: analetheism, contraction, dialetheism, naive truth, restricted quantification, substructural logics, transitivity

(C) 2016 by University of Notre Dame $\quad$ 10.1215/00294527-3429057 


\title{
Restriction by Noncontraction
}

\author{
Elia Zardini
}

\begin{abstract}
This paper investigates how naive theories of truth fare with respect to a set of extremely plausible principles of restricted quantification. It is first shown that both nonsubstructural theories as well as certain substructural theories cannot validate all those principles. Then, pursuing further an approach to the semantic paradoxes that the author has defended elsewhere, the theory of restricted quantification available in a specific naive theory that rejects the structural property of contraction is explored. It is shown that the theory validates all the principles in question, and it is argued that other prima facie plausible principles that the theory fails to validate are objectionable on independent grounds.
\end{abstract}

\section{Implication}

An operation of implication $^{1}$ has traditionally been associated with at least six different (possibly related) functions (which I will refer to as 'the Functions'). Taking an implicational proposition expressed by the conditional $\varphi \rightarrow \psi$, these can informally be expressed as:

SUFFICIENT CONDITION $\Rightarrow$ : Be at least as weak as $\varphi$ being a sufficient condition for $\psi$;

SUFFICIENT CONDITION ${ }^{\Leftarrow}$ : Be at least as strong as $\varphi$ being a sufficient condition for $\psi$;

NO REFUTATION $\Rightarrow$ : Follow from its not being the case that $\varphi$ holds while $\psi$ does not; ${ }^{2}$

NO REFUTATION ${ }^{\leftarrow}$ : Entail that it is not the case that $\varphi$ holds while $\psi$ does not;

DEDUCTION THEOREM: Be at least as weak as $\varphi$ entailing $\psi$;

MODUS PONENS: Together with $\varphi$, logically necessitate $\psi$. $^{3}$

Received September 1, 2012; accepted October 14, 2013

First published online January 28, 2016

2010 Mathematics Subject Classification: Primary 03; Secondary A05, B20, B47, B53, B65

Keywords: analetheism, contraction, dialetheism, naive truth, restricted quantification, substructural logics, transitivity

(C) 2016 by University of Notre Dame $\quad$ 10.1215/00294527-3429057 


\title{
Restriction by Noncontraction
}

\author{
Elia Zardini
}

\begin{abstract}
This paper investigates how naive theories of truth fare with respect to a set of extremely plausible principles of restricted quantification. It is first shown that both nonsubstructural theories as well as certain substructural theories cannot validate all those principles. Then, pursuing further an approach to the semantic paradoxes that the author has defended elsewhere, the theory of restricted quantification available in a specific naive theory that rejects the structural property of contraction is explored. It is shown that the theory validates all the principles in question, and it is argued that other prima facie plausible principles that the theory fails to validate are objectionable on independent grounds.
\end{abstract}

\section{Implication}

An operation of implication $^{1}$ has traditionally been associated with at least six different (possibly related) functions (which I will refer to as 'the Functions'). Taking an implicational proposition expressed by the conditional $\varphi \rightarrow \psi$, these can informally be expressed as:

SUFFICIENT CONDITION $\Rightarrow$ : Be at least as weak as $\varphi$ being a sufficient condition for $\psi$;

SUFFICIENT CONDITION ${ }^{\Leftarrow}$ : Be at least as strong as $\varphi$ being a sufficient condition for $\psi$;

NO REFUTATION $\Rightarrow$ : Follow from its not being the case that $\varphi$ holds while $\psi$ does not; ${ }^{2}$

NO REFUTATION ${ }^{\leftarrow}$ : Entail that it is not the case that $\varphi$ holds while $\psi$ does not;

DEDUCTION THEOREM: Be at least as weak as $\varphi$ entailing $\psi$;

MODUS PONENS: Together with $\varphi$, logically necessitate $\psi$. $^{3}$

Received September 1, 2012; accepted October 14, 2013

First published online January 28, 2016

2010 Mathematics Subject Classification: Primary 03; Secondary A05, B20, B47, B53, B65

Keywords: analetheism, contraction, dialetheism, naive truth, restricted quantification, substructural logics, transitivity

(C) 2016 by University of Notre Dame $\quad$ 10.1215/00294527-3429057 


\title{
Restriction by Noncontraction
}

\author{
Elia Zardini
}

\begin{abstract}
This paper investigates how naive theories of truth fare with respect to a set of extremely plausible principles of restricted quantification. It is first shown that both nonsubstructural theories as well as certain substructural theories cannot validate all those principles. Then, pursuing further an approach to the semantic paradoxes that the author has defended elsewhere, the theory of restricted quantification available in a specific naive theory that rejects the structural property of contraction is explored. It is shown that the theory validates all the principles in question, and it is argued that other prima facie plausible principles that the theory fails to validate are objectionable on independent grounds.
\end{abstract}

\section{Implication}

An operation of implication $^{1}$ has traditionally been associated with at least six different (possibly related) functions (which I will refer to as 'the Functions'). Taking an implicational proposition expressed by the conditional $\varphi \rightarrow \psi$, these can informally be expressed as:

SUFFICIENT CONDITION $\Rightarrow$ : Be at least as weak as $\varphi$ being a sufficient condition for $\psi$;

SUFFICIENT CONDITION ${ }^{\Leftarrow}$ : Be at least as strong as $\varphi$ being a sufficient condition for $\psi$;

NO REFUTATION $\Rightarrow$ : Follow from its not being the case that $\varphi$ holds while $\psi$ does not; ${ }^{2}$

NO REFUTATION ${ }^{\leftarrow}$ : Entail that it is not the case that $\varphi$ holds while $\psi$ does not;

DEDUCTION THEOREM: Be at least as weak as $\varphi$ entailing $\psi$;

MODUS PONENS: Together with $\varphi$, logically necessitate $\psi$. $^{3}$

Received September 1, 2012; accepted October 14, 2013

First published online January 28, 2016

2010 Mathematics Subject Classification: Primary 03; Secondary A05, B20, B47, B53, B65

Keywords: analetheism, contraction, dialetheism, naive truth, restricted quantification, substructural logics, transitivity

(C) 2016 by University of Notre Dame $\quad$ 10.1215/00294527-3429057 


\title{
Restriction by Noncontraction
}

\author{
Elia Zardini
}

\begin{abstract}
This paper investigates how naive theories of truth fare with respect to a set of extremely plausible principles of restricted quantification. It is first shown that both nonsubstructural theories as well as certain substructural theories cannot validate all those principles. Then, pursuing further an approach to the semantic paradoxes that the author has defended elsewhere, the theory of restricted quantification available in a specific naive theory that rejects the structural property of contraction is explored. It is shown that the theory validates all the principles in question, and it is argued that other prima facie plausible principles that the theory fails to validate are objectionable on independent grounds.
\end{abstract}

\section{Implication}

An operation of implication $^{1}$ has traditionally been associated with at least six different (possibly related) functions (which I will refer to as 'the Functions'). Taking an implicational proposition expressed by the conditional $\varphi \rightarrow \psi$, these can informally be expressed as:

SUFFICIENT CONDITION $\Rightarrow$ : Be at least as weak as $\varphi$ being a sufficient condition for $\psi$;

SUFFICIENT CONDITION ${ }^{\Leftarrow}$ : Be at least as strong as $\varphi$ being a sufficient condition for $\psi$;

NO REFUTATION $\Rightarrow$ : Follow from its not being the case that $\varphi$ holds while $\psi$ does not; ${ }^{2}$

NO REFUTATION ${ }^{\leftarrow}$ : Entail that it is not the case that $\varphi$ holds while $\psi$ does not;

DEDUCTION THEOREM: Be at least as weak as $\varphi$ entailing $\psi$;

MODUS PONENS: Together with $\varphi$, logically necessitate $\psi$. $^{3}$

Received September 1, 2012; accepted October 14, 2013

First published online January 28, 2016

2010 Mathematics Subject Classification: Primary 03; Secondary A05, B20, B47, B53, B65

Keywords: analetheism, contraction, dialetheism, naive truth, restricted quantification, substructural logics, transitivity

(C) 2016 by University of Notre Dame $\quad$ 10.1215/00294527-3429057 


\title{
Restriction by Noncontraction
}

\author{
Elia Zardini
}

\begin{abstract}
This paper investigates how naive theories of truth fare with respect to a set of extremely plausible principles of restricted quantification. It is first shown that both nonsubstructural theories as well as certain substructural theories cannot validate all those principles. Then, pursuing further an approach to the semantic paradoxes that the author has defended elsewhere, the theory of restricted quantification available in a specific naive theory that rejects the structural property of contraction is explored. It is shown that the theory validates all the principles in question, and it is argued that other prima facie plausible principles that the theory fails to validate are objectionable on independent grounds.
\end{abstract}

\section{Implication}

An operation of implication $^{1}$ has traditionally been associated with at least six different (possibly related) functions (which I will refer to as 'the Functions'). Taking an implicational proposition expressed by the conditional $\varphi \rightarrow \psi$, these can informally be expressed as:

SUFFICIENT CONDITION $\Rightarrow$ : Be at least as weak as $\varphi$ being a sufficient condition for $\psi$;

SUFFICIENT CONDITION ${ }^{\Leftarrow}$ : Be at least as strong as $\varphi$ being a sufficient condition for $\psi$;

NO REFUTATION $\Rightarrow$ : Follow from its not being the case that $\varphi$ holds while $\psi$ does not; ${ }^{2}$

NO REFUTATION ${ }^{\leftarrow}$ : Entail that it is not the case that $\varphi$ holds while $\psi$ does not;

DEDUCTION THEOREM: Be at least as weak as $\varphi$ entailing $\psi$;

MODUS PONENS: Together with $\varphi$, logically necessitate $\psi$. $^{3}$

Received September 1, 2012; accepted October 14, 2013

First published online January 28, 2016

2010 Mathematics Subject Classification: Primary 03; Secondary A05, B20, B47, B53, B65

Keywords: analetheism, contraction, dialetheism, naive truth, restricted quantification, substructural logics, transitivity

(C) 2016 by University of Notre Dame $\quad$ 10.1215/00294527-3429057 


\title{
Restriction by Noncontraction
}

\author{
Elia Zardini
}

\begin{abstract}
This paper investigates how naive theories of truth fare with respect to a set of extremely plausible principles of restricted quantification. It is first shown that both nonsubstructural theories as well as certain substructural theories cannot validate all those principles. Then, pursuing further an approach to the semantic paradoxes that the author has defended elsewhere, the theory of restricted quantification available in a specific naive theory that rejects the structural property of contraction is explored. It is shown that the theory validates all the principles in question, and it is argued that other prima facie plausible principles that the theory fails to validate are objectionable on independent grounds.
\end{abstract}

\section{Implication}

An operation of implication $^{1}$ has traditionally been associated with at least six different (possibly related) functions (which I will refer to as 'the Functions'). Taking an implicational proposition expressed by the conditional $\varphi \rightarrow \psi$, these can informally be expressed as:

SUFFICIENT CONDITION $\Rightarrow$ : Be at least as weak as $\varphi$ being a sufficient condition for $\psi$;

SUFFICIENT CONDITION ${ }^{\Leftarrow}$ : Be at least as strong as $\varphi$ being a sufficient condition for $\psi$;

NO REFUTATION $\Rightarrow$ : Follow from its not being the case that $\varphi$ holds while $\psi$ does not; ${ }^{2}$

NO REFUTATION ${ }^{\leftarrow}$ : Entail that it is not the case that $\varphi$ holds while $\psi$ does not;

DEDUCTION THEOREM: Be at least as weak as $\varphi$ entailing $\psi$;

MODUS PONENS: Together with $\varphi$, logically necessitate $\psi$. $^{3}$

Received September 1, 2012; accepted October 14, 2013

First published online January 28, 2016

2010 Mathematics Subject Classification: Primary 03; Secondary A05, B20, B47, B53, B65

Keywords: analetheism, contraction, dialetheism, naive truth, restricted quantification, substructural logics, transitivity

(C) 2016 by University of Notre Dame $\quad$ 10.1215/00294527-3429057 


\title{
Restriction by Noncontraction
}

\author{
Elia Zardini
}

\begin{abstract}
This paper investigates how naive theories of truth fare with respect to a set of extremely plausible principles of restricted quantification. It is first shown that both nonsubstructural theories as well as certain substructural theories cannot validate all those principles. Then, pursuing further an approach to the semantic paradoxes that the author has defended elsewhere, the theory of restricted quantification available in a specific naive theory that rejects the structural property of contraction is explored. It is shown that the theory validates all the principles in question, and it is argued that other prima facie plausible principles that the theory fails to validate are objectionable on independent grounds.
\end{abstract}

\section{Implication}

An operation of implication $^{1}$ has traditionally been associated with at least six different (possibly related) functions (which I will refer to as 'the Functions'). Taking an implicational proposition expressed by the conditional $\varphi \rightarrow \psi$, these can informally be expressed as:

SUFFICIENT CONDITION $\Rightarrow$ : Be at least as weak as $\varphi$ being a sufficient condition for $\psi$;

SUFFICIENT CONDITION ${ }^{\Leftarrow}$ : Be at least as strong as $\varphi$ being a sufficient condition for $\psi$;

NO REFUTATION $\Rightarrow$ : Follow from its not being the case that $\varphi$ holds while $\psi$ does not; ${ }^{2}$

NO REFUTATION ${ }^{\leftarrow}$ : Entail that it is not the case that $\varphi$ holds while $\psi$ does not;

DEDUCTION THEOREM: Be at least as weak as $\varphi$ entailing $\psi$;

MODUS PONENS: Together with $\varphi$, logically necessitate $\psi$. $^{3}$

Received September 1, 2012; accepted October 14, 2013

First published online January 28, 2016

2010 Mathematics Subject Classification: Primary 03; Secondary A05, B20, B47, B53, B65

Keywords: analetheism, contraction, dialetheism, naive truth, restricted quantification, substructural logics, transitivity

(C) 2016 by University of Notre Dame $\quad$ 10.1215/00294527-3429057 


\title{
Restriction by Noncontraction
}

\author{
Elia Zardini
}

\begin{abstract}
This paper investigates how naive theories of truth fare with respect to a set of extremely plausible principles of restricted quantification. It is first shown that both nonsubstructural theories as well as certain substructural theories cannot validate all those principles. Then, pursuing further an approach to the semantic paradoxes that the author has defended elsewhere, the theory of restricted quantification available in a specific naive theory that rejects the structural property of contraction is explored. It is shown that the theory validates all the principles in question, and it is argued that other prima facie plausible principles that the theory fails to validate are objectionable on independent grounds.
\end{abstract}

\section{Implication}

An operation of implication $^{1}$ has traditionally been associated with at least six different (possibly related) functions (which I will refer to as 'the Functions'). Taking an implicational proposition expressed by the conditional $\varphi \rightarrow \psi$, these can informally be expressed as:

SUFFICIENT CONDITION $\Rightarrow$ : Be at least as weak as $\varphi$ being a sufficient condition for $\psi$;

SUFFICIENT CONDITION ${ }^{\Leftarrow}$ : Be at least as strong as $\varphi$ being a sufficient condition for $\psi$;

NO REFUTATION $\Rightarrow$ : Follow from its not being the case that $\varphi$ holds while $\psi$ does not; ${ }^{2}$

NO REFUTATION ${ }^{\leftarrow}$ : Entail that it is not the case that $\varphi$ holds while $\psi$ does not;

DEDUCTION THEOREM: Be at least as weak as $\varphi$ entailing $\psi$;

MODUS PONENS: Together with $\varphi$, logically necessitate $\psi$. $^{3}$

Received September 1, 2012; accepted October 14, 2013

First published online January 28, 2016

2010 Mathematics Subject Classification: Primary 03; Secondary A05, B20, B47, B53, B65

Keywords: analetheism, contraction, dialetheism, naive truth, restricted quantification, substructural logics, transitivity

(C) 2016 by University of Notre Dame $\quad$ 10.1215/00294527-3429057 


\title{
Restriction by Noncontraction
}

\author{
Elia Zardini
}

\begin{abstract}
This paper investigates how naive theories of truth fare with respect to a set of extremely plausible principles of restricted quantification. It is first shown that both nonsubstructural theories as well as certain substructural theories cannot validate all those principles. Then, pursuing further an approach to the semantic paradoxes that the author has defended elsewhere, the theory of restricted quantification available in a specific naive theory that rejects the structural property of contraction is explored. It is shown that the theory validates all the principles in question, and it is argued that other prima facie plausible principles that the theory fails to validate are objectionable on independent grounds.
\end{abstract}

\section{Implication}

An operation of implication $^{1}$ has traditionally been associated with at least six different (possibly related) functions (which I will refer to as 'the Functions'). Taking an implicational proposition expressed by the conditional $\varphi \rightarrow \psi$, these can informally be expressed as:

SUFFICIENT CONDITION $\Rightarrow$ : Be at least as weak as $\varphi$ being a sufficient condition for $\psi$;

SUFFICIENT CONDITION ${ }^{\Leftarrow}$ : Be at least as strong as $\varphi$ being a sufficient condition for $\psi$;

NO REFUTATION $\Rightarrow$ : Follow from its not being the case that $\varphi$ holds while $\psi$ does not; ${ }^{2}$

NO REFUTATION ${ }^{\leftarrow}$ : Entail that it is not the case that $\varphi$ holds while $\psi$ does not;

DEDUCTION THEOREM: Be at least as weak as $\varphi$ entailing $\psi$;

MODUS PONENS: Together with $\varphi$, logically necessitate $\psi$. $^{3}$

Received September 1, 2012; accepted October 14, 2013

First published online January 28, 2016

2010 Mathematics Subject Classification: Primary 03; Secondary A05, B20, B47, B53, B65

Keywords: analetheism, contraction, dialetheism, naive truth, restricted quantification, substructural logics, transitivity

(C) 2016 by University of Notre Dame $\quad$ 10.1215/00294527-3429057 


\title{
Restriction by Noncontraction
}

\author{
Elia Zardini
}

\begin{abstract}
This paper investigates how naive theories of truth fare with respect to a set of extremely plausible principles of restricted quantification. It is first shown that both nonsubstructural theories as well as certain substructural theories cannot validate all those principles. Then, pursuing further an approach to the semantic paradoxes that the author has defended elsewhere, the theory of restricted quantification available in a specific naive theory that rejects the structural property of contraction is explored. It is shown that the theory validates all the principles in question, and it is argued that other prima facie plausible principles that the theory fails to validate are objectionable on independent grounds.
\end{abstract}

\section{Implication}

An operation of implication $^{1}$ has traditionally been associated with at least six different (possibly related) functions (which I will refer to as 'the Functions'). Taking an implicational proposition expressed by the conditional $\varphi \rightarrow \psi$, these can informally be expressed as:

SUFFICIENT CONDITION $\Rightarrow$ : Be at least as weak as $\varphi$ being a sufficient condition for $\psi$;

SUFFICIENT CONDITION ${ }^{\Leftarrow}$ : Be at least as strong as $\varphi$ being a sufficient condition for $\psi$;

NO REFUTATION $\Rightarrow$ : Follow from its not being the case that $\varphi$ holds while $\psi$ does not; ${ }^{2}$

NO REFUTATION ${ }^{\leftarrow}$ : Entail that it is not the case that $\varphi$ holds while $\psi$ does not;

DEDUCTION THEOREM: Be at least as weak as $\varphi$ entailing $\psi$;

MODUS PONENS: Together with $\varphi$, logically necessitate $\psi$. $^{3}$

Received September 1, 2012; accepted October 14, 2013

First published online January 28, 2016

2010 Mathematics Subject Classification: Primary 03; Secondary A05, B20, B47, B53, B65

Keywords: analetheism, contraction, dialetheism, naive truth, restricted quantification, substructural logics, transitivity

(C) 2016 by University of Notre Dame $\quad$ 10.1215/00294527-3429057 


\title{
Restriction by Noncontraction
}

\author{
Elia Zardini
}

\begin{abstract}
This paper investigates how naive theories of truth fare with respect to a set of extremely plausible principles of restricted quantification. It is first shown that both nonsubstructural theories as well as certain substructural theories cannot validate all those principles. Then, pursuing further an approach to the semantic paradoxes that the author has defended elsewhere, the theory of restricted quantification available in a specific naive theory that rejects the structural property of contraction is explored. It is shown that the theory validates all the principles in question, and it is argued that other prima facie plausible principles that the theory fails to validate are objectionable on independent grounds.
\end{abstract}

\section{Implication}

An operation of implication $^{1}$ has traditionally been associated with at least six different (possibly related) functions (which I will refer to as 'the Functions'). Taking an implicational proposition expressed by the conditional $\varphi \rightarrow \psi$, these can informally be expressed as:

SUFFICIENT CONDITION $\Rightarrow$ : Be at least as weak as $\varphi$ being a sufficient condition for $\psi$;

SUFFICIENT CONDITION ${ }^{\Leftarrow}$ : Be at least as strong as $\varphi$ being a sufficient condition for $\psi$;

NO REFUTATION $\Rightarrow$ : Follow from its not being the case that $\varphi$ holds while $\psi$ does not; ${ }^{2}$

NO REFUTATION ${ }^{\leftarrow}$ : Entail that it is not the case that $\varphi$ holds while $\psi$ does not;

DEDUCTION THEOREM: Be at least as weak as $\varphi$ entailing $\psi$;

MODUS PONENS: Together with $\varphi$, logically necessitate $\psi$. $^{3}$

Received September 1, 2012; accepted October 14, 2013

First published online January 28, 2016

2010 Mathematics Subject Classification: Primary 03; Secondary A05, B20, B47, B53, B65

Keywords: analetheism, contraction, dialetheism, naive truth, restricted quantification, substructural logics, transitivity

(C) 2016 by University of Notre Dame $\quad$ 10.1215/00294527-3429057 


\title{
Restriction by Noncontraction
}

\author{
Elia Zardini
}

\begin{abstract}
This paper investigates how naive theories of truth fare with respect to a set of extremely plausible principles of restricted quantification. It is first shown that both nonsubstructural theories as well as certain substructural theories cannot validate all those principles. Then, pursuing further an approach to the semantic paradoxes that the author has defended elsewhere, the theory of restricted quantification available in a specific naive theory that rejects the structural property of contraction is explored. It is shown that the theory validates all the principles in question, and it is argued that other prima facie plausible principles that the theory fails to validate are objectionable on independent grounds.
\end{abstract}

\section{Implication}

An operation of implication $^{1}$ has traditionally been associated with at least six different (possibly related) functions (which I will refer to as 'the Functions'). Taking an implicational proposition expressed by the conditional $\varphi \rightarrow \psi$, these can informally be expressed as:

SUFFICIENT CONDITION $\Rightarrow$ : Be at least as weak as $\varphi$ being a sufficient condition for $\psi$;

SUFFICIENT CONDITION ${ }^{\Leftarrow}$ : Be at least as strong as $\varphi$ being a sufficient condition for $\psi$;

NO REFUTATION $\Rightarrow$ : Follow from its not being the case that $\varphi$ holds while $\psi$ does not; ${ }^{2}$

NO REFUTATION ${ }^{\leftarrow}$ : Entail that it is not the case that $\varphi$ holds while $\psi$ does not;

DEDUCTION THEOREM: Be at least as weak as $\varphi$ entailing $\psi$;

MODUS PONENS: Together with $\varphi$, logically necessitate $\psi$. $^{3}$

Received September 1, 2012; accepted October 14, 2013

First published online January 28, 2016

2010 Mathematics Subject Classification: Primary 03; Secondary A05, B20, B47, B53, B65

Keywords: analetheism, contraction, dialetheism, naive truth, restricted quantification, substructural logics, transitivity

(C) 2016 by University of Notre Dame $\quad$ 10.1215/00294527-3429057 


\title{
Restriction by Noncontraction
}

\author{
Elia Zardini
}

\begin{abstract}
This paper investigates how naive theories of truth fare with respect to a set of extremely plausible principles of restricted quantification. It is first shown that both nonsubstructural theories as well as certain substructural theories cannot validate all those principles. Then, pursuing further an approach to the semantic paradoxes that the author has defended elsewhere, the theory of restricted quantification available in a specific naive theory that rejects the structural property of contraction is explored. It is shown that the theory validates all the principles in question, and it is argued that other prima facie plausible principles that the theory fails to validate are objectionable on independent grounds.
\end{abstract}

\section{Implication}

An operation of implication $^{1}$ has traditionally been associated with at least six different (possibly related) functions (which I will refer to as 'the Functions'). Taking an implicational proposition expressed by the conditional $\varphi \rightarrow \psi$, these can informally be expressed as:

SUFFICIENT CONDITION $\Rightarrow$ : Be at least as weak as $\varphi$ being a sufficient condition for $\psi$;

SUFFICIENT CONDITION ${ }^{\Leftarrow}$ : Be at least as strong as $\varphi$ being a sufficient condition for $\psi$;

NO REFUTATION $\Rightarrow$ : Follow from its not being the case that $\varphi$ holds while $\psi$ does not; ${ }^{2}$

NO REFUTATION ${ }^{\leftarrow}$ : Entail that it is not the case that $\varphi$ holds while $\psi$ does not;

DEDUCTION THEOREM: Be at least as weak as $\varphi$ entailing $\psi$;

MODUS PONENS: Together with $\varphi$, logically necessitate $\psi$. $^{3}$

Received September 1, 2012; accepted October 14, 2013

First published online January 28, 2016

2010 Mathematics Subject Classification: Primary 03; Secondary A05, B20, B47, B53, B65

Keywords: analetheism, contraction, dialetheism, naive truth, restricted quantification, substructural logics, transitivity

(C) 2016 by University of Notre Dame $\quad$ 10.1215/00294527-3429057 


\title{
Restriction by Noncontraction
}

\author{
Elia Zardini
}

\begin{abstract}
This paper investigates how naive theories of truth fare with respect to a set of extremely plausible principles of restricted quantification. It is first shown that both nonsubstructural theories as well as certain substructural theories cannot validate all those principles. Then, pursuing further an approach to the semantic paradoxes that the author has defended elsewhere, the theory of restricted quantification available in a specific naive theory that rejects the structural property of contraction is explored. It is shown that the theory validates all the principles in question, and it is argued that other prima facie plausible principles that the theory fails to validate are objectionable on independent grounds.
\end{abstract}

\section{Implication}

An operation of implication $^{1}$ has traditionally been associated with at least six different (possibly related) functions (which I will refer to as 'the Functions'). Taking an implicational proposition expressed by the conditional $\varphi \rightarrow \psi$, these can informally be expressed as:

SUFFICIENT CONDITION $\Rightarrow$ : Be at least as weak as $\varphi$ being a sufficient condition for $\psi$;

SUFFICIENT CONDITION ${ }^{\Leftarrow}$ : Be at least as strong as $\varphi$ being a sufficient condition for $\psi$;

NO REFUTATION $\Rightarrow$ : Follow from its not being the case that $\varphi$ holds while $\psi$ does not; ${ }^{2}$

NO REFUTATION ${ }^{\leftarrow}$ : Entail that it is not the case that $\varphi$ holds while $\psi$ does not;

DEDUCTION THEOREM: Be at least as weak as $\varphi$ entailing $\psi$;

MODUS PONENS: Together with $\varphi$, logically necessitate $\psi$. $^{3}$

Received September 1, 2012; accepted October 14, 2013

First published online January 28, 2016

2010 Mathematics Subject Classification: Primary 03; Secondary A05, B20, B47, B53, B65

Keywords: analetheism, contraction, dialetheism, naive truth, restricted quantification, substructural logics, transitivity

(C) 2016 by University of Notre Dame $\quad$ 10.1215/00294527-3429057 


\title{
Restriction by Noncontraction
}

\author{
Elia Zardini
}

\begin{abstract}
This paper investigates how naive theories of truth fare with respect to a set of extremely plausible principles of restricted quantification. It is first shown that both nonsubstructural theories as well as certain substructural theories cannot validate all those principles. Then, pursuing further an approach to the semantic paradoxes that the author has defended elsewhere, the theory of restricted quantification available in a specific naive theory that rejects the structural property of contraction is explored. It is shown that the theory validates all the principles in question, and it is argued that other prima facie plausible principles that the theory fails to validate are objectionable on independent grounds.
\end{abstract}

\section{Implication}

An operation of implication $^{1}$ has traditionally been associated with at least six different (possibly related) functions (which I will refer to as 'the Functions'). Taking an implicational proposition expressed by the conditional $\varphi \rightarrow \psi$, these can informally be expressed as:

SUFFICIENT CONDITION $\Rightarrow$ : Be at least as weak as $\varphi$ being a sufficient condition for $\psi$;

SUFFICIENT CONDITION ${ }^{\Leftarrow}$ : Be at least as strong as $\varphi$ being a sufficient condition for $\psi$;

NO REFUTATION $\Rightarrow$ : Follow from its not being the case that $\varphi$ holds while $\psi$ does not; ${ }^{2}$

NO REFUTATION ${ }^{\leftarrow}$ : Entail that it is not the case that $\varphi$ holds while $\psi$ does not;

DEDUCTION THEOREM: Be at least as weak as $\varphi$ entailing $\psi$;

MODUS PONENS: Together with $\varphi$, logically necessitate $\psi$. $^{3}$

Received September 1, 2012; accepted October 14, 2013

First published online January 28, 2016

2010 Mathematics Subject Classification: Primary 03; Secondary A05, B20, B47, B53, B65

Keywords: analetheism, contraction, dialetheism, naive truth, restricted quantification, substructural logics, transitivity

(C) 2016 by University of Notre Dame $\quad$ 10.1215/00294527-3429057 


\title{
Restriction by Noncontraction
}

\author{
Elia Zardini
}

\begin{abstract}
This paper investigates how naive theories of truth fare with respect to a set of extremely plausible principles of restricted quantification. It is first shown that both nonsubstructural theories as well as certain substructural theories cannot validate all those principles. Then, pursuing further an approach to the semantic paradoxes that the author has defended elsewhere, the theory of restricted quantification available in a specific naive theory that rejects the structural property of contraction is explored. It is shown that the theory validates all the principles in question, and it is argued that other prima facie plausible principles that the theory fails to validate are objectionable on independent grounds.
\end{abstract}

\section{Implication}

An operation of implication $^{1}$ has traditionally been associated with at least six different (possibly related) functions (which I will refer to as 'the Functions'). Taking an implicational proposition expressed by the conditional $\varphi \rightarrow \psi$, these can informally be expressed as:

SUFFICIENT CONDITION $\Rightarrow$ : Be at least as weak as $\varphi$ being a sufficient condition for $\psi$;

SUFFICIENT CONDITION ${ }^{\Leftarrow}$ : Be at least as strong as $\varphi$ being a sufficient condition for $\psi$;

NO REFUTATION $\Rightarrow$ : Follow from its not being the case that $\varphi$ holds while $\psi$ does not; ${ }^{2}$

NO REFUTATION ${ }^{\leftarrow}$ : Entail that it is not the case that $\varphi$ holds while $\psi$ does not;

DEDUCTION THEOREM: Be at least as weak as $\varphi$ entailing $\psi$;

MODUS PONENS: Together with $\varphi$, logically necessitate $\psi$. $^{3}$

Received September 1, 2012; accepted October 14, 2013

First published online January 28, 2016

2010 Mathematics Subject Classification: Primary 03; Secondary A05, B20, B47, B53, B65

Keywords: analetheism, contraction, dialetheism, naive truth, restricted quantification, substructural logics, transitivity

(C) 2016 by University of Notre Dame $\quad$ 10.1215/00294527-3429057 


\title{
Restriction by Noncontraction
}

\author{
Elia Zardini
}

\begin{abstract}
This paper investigates how naive theories of truth fare with respect to a set of extremely plausible principles of restricted quantification. It is first shown that both nonsubstructural theories as well as certain substructural theories cannot validate all those principles. Then, pursuing further an approach to the semantic paradoxes that the author has defended elsewhere, the theory of restricted quantification available in a specific naive theory that rejects the structural property of contraction is explored. It is shown that the theory validates all the principles in question, and it is argued that other prima facie plausible principles that the theory fails to validate are objectionable on independent grounds.
\end{abstract}

\section{Implication}

An operation of implication $^{1}$ has traditionally been associated with at least six different (possibly related) functions (which I will refer to as 'the Functions'). Taking an implicational proposition expressed by the conditional $\varphi \rightarrow \psi$, these can informally be expressed as:

SUFFICIENT CONDITION $\Rightarrow$ : Be at least as weak as $\varphi$ being a sufficient condition for $\psi$;

SUFFICIENT CONDITION ${ }^{\Leftarrow}$ : Be at least as strong as $\varphi$ being a sufficient condition for $\psi$;

NO REFUTATION $\Rightarrow$ : Follow from its not being the case that $\varphi$ holds while $\psi$ does not; ${ }^{2}$

NO REFUTATION ${ }^{\leftarrow}$ : Entail that it is not the case that $\varphi$ holds while $\psi$ does not;

DEDUCTION THEOREM: Be at least as weak as $\varphi$ entailing $\psi$;

MODUS PONENS: Together with $\varphi$, logically necessitate $\psi$. $^{3}$

Received September 1, 2012; accepted October 14, 2013

First published online January 28, 2016

2010 Mathematics Subject Classification: Primary 03; Secondary A05, B20, B47, B53, B65

Keywords: analetheism, contraction, dialetheism, naive truth, restricted quantification, substructural logics, transitivity

(C) 2016 by University of Notre Dame $\quad$ 10.1215/00294527-3429057 


\title{
Restriction by Noncontraction
}

\author{
Elia Zardini
}

\begin{abstract}
This paper investigates how naive theories of truth fare with respect to a set of extremely plausible principles of restricted quantification. It is first shown that both nonsubstructural theories as well as certain substructural theories cannot validate all those principles. Then, pursuing further an approach to the semantic paradoxes that the author has defended elsewhere, the theory of restricted quantification available in a specific naive theory that rejects the structural property of contraction is explored. It is shown that the theory validates all the principles in question, and it is argued that other prima facie plausible principles that the theory fails to validate are objectionable on independent grounds.
\end{abstract}

\section{Implication}

An operation of implication $^{1}$ has traditionally been associated with at least six different (possibly related) functions (which I will refer to as 'the Functions'). Taking an implicational proposition expressed by the conditional $\varphi \rightarrow \psi$, these can informally be expressed as:

SUFFICIENT CONDITION $\Rightarrow$ : Be at least as weak as $\varphi$ being a sufficient condition for $\psi$;

SUFFICIENT CONDITION ${ }^{\Leftarrow}$ : Be at least as strong as $\varphi$ being a sufficient condition for $\psi$;

NO REFUTATION $\Rightarrow$ : Follow from its not being the case that $\varphi$ holds while $\psi$ does not; ${ }^{2}$

NO REFUTATION ${ }^{\leftarrow}$ : Entail that it is not the case that $\varphi$ holds while $\psi$ does not;

DEDUCTION THEOREM: Be at least as weak as $\varphi$ entailing $\psi$;

MODUS PONENS: Together with $\varphi$, logically necessitate $\psi$. $^{3}$

Received September 1, 2012; accepted October 14, 2013

First published online January 28, 2016

2010 Mathematics Subject Classification: Primary 03; Secondary A05, B20, B47, B53, B65

Keywords: analetheism, contraction, dialetheism, naive truth, restricted quantification, substructural logics, transitivity

(C) 2016 by University of Notre Dame $\quad$ 10.1215/00294527-3429057 


\title{
Restriction by Noncontraction
}

\author{
Elia Zardini
}

\begin{abstract}
This paper investigates how naive theories of truth fare with respect to a set of extremely plausible principles of restricted quantification. It is first shown that both nonsubstructural theories as well as certain substructural theories cannot validate all those principles. Then, pursuing further an approach to the semantic paradoxes that the author has defended elsewhere, the theory of restricted quantification available in a specific naive theory that rejects the structural property of contraction is explored. It is shown that the theory validates all the principles in question, and it is argued that other prima facie plausible principles that the theory fails to validate are objectionable on independent grounds.
\end{abstract}

\section{Implication}

An operation of implication $^{1}$ has traditionally been associated with at least six different (possibly related) functions (which I will refer to as 'the Functions'). Taking an implicational proposition expressed by the conditional $\varphi \rightarrow \psi$, these can informally be expressed as:

SUFFICIENT CONDITION $\Rightarrow$ : Be at least as weak as $\varphi$ being a sufficient condition for $\psi$;

SUFFICIENT CONDITION ${ }^{\Leftarrow}$ : Be at least as strong as $\varphi$ being a sufficient condition for $\psi$;

NO REFUTATION $\Rightarrow$ : Follow from its not being the case that $\varphi$ holds while $\psi$ does not; ${ }^{2}$

NO REFUTATION ${ }^{\leftarrow}$ : Entail that it is not the case that $\varphi$ holds while $\psi$ does not;

DEDUCTION THEOREM: Be at least as weak as $\varphi$ entailing $\psi$;

MODUS PONENS: Together with $\varphi$, logically necessitate $\psi$. $^{3}$

Received September 1, 2012; accepted October 14, 2013

First published online January 28, 2016

2010 Mathematics Subject Classification: Primary 03; Secondary A05, B20, B47, B53, B65

Keywords: analetheism, contraction, dialetheism, naive truth, restricted quantification, substructural logics, transitivity

(C) 2016 by University of Notre Dame $\quad$ 10.1215/00294527-3429057 


\title{
Restriction by Noncontraction
}

\author{
Elia Zardini
}

\begin{abstract}
This paper investigates how naive theories of truth fare with respect to a set of extremely plausible principles of restricted quantification. It is first shown that both nonsubstructural theories as well as certain substructural theories cannot validate all those principles. Then, pursuing further an approach to the semantic paradoxes that the author has defended elsewhere, the theory of restricted quantification available in a specific naive theory that rejects the structural property of contraction is explored. It is shown that the theory validates all the principles in question, and it is argued that other prima facie plausible principles that the theory fails to validate are objectionable on independent grounds.
\end{abstract}

\section{Implication}

An operation of implication $^{1}$ has traditionally been associated with at least six different (possibly related) functions (which I will refer to as 'the Functions'). Taking an implicational proposition expressed by the conditional $\varphi \rightarrow \psi$, these can informally be expressed as:

SUFFICIENT CONDITION $\Rightarrow$ : Be at least as weak as $\varphi$ being a sufficient condition for $\psi$;

SUFFICIENT CONDITION ${ }^{\Leftarrow}$ : Be at least as strong as $\varphi$ being a sufficient condition for $\psi$;

NO REFUTATION $\Rightarrow$ : Follow from its not being the case that $\varphi$ holds while $\psi$ does not; ${ }^{2}$

NO REFUTATION ${ }^{\leftarrow}$ : Entail that it is not the case that $\varphi$ holds while $\psi$ does not;

DEDUCTION THEOREM: Be at least as weak as $\varphi$ entailing $\psi$;

MODUS PONENS: Together with $\varphi$, logically necessitate $\psi$. $^{3}$

Received September 1, 2012; accepted October 14, 2013

First published online January 28, 2016

2010 Mathematics Subject Classification: Primary 03; Secondary A05, B20, B47, B53, B65

Keywords: analetheism, contraction, dialetheism, naive truth, restricted quantification, substructural logics, transitivity

(C) 2016 by University of Notre Dame $\quad$ 10.1215/00294527-3429057 


\title{
Restriction by Noncontraction
}

\author{
Elia Zardini
}

\begin{abstract}
This paper investigates how naive theories of truth fare with respect to a set of extremely plausible principles of restricted quantification. It is first shown that both nonsubstructural theories as well as certain substructural theories cannot validate all those principles. Then, pursuing further an approach to the semantic paradoxes that the author has defended elsewhere, the theory of restricted quantification available in a specific naive theory that rejects the structural property of contraction is explored. It is shown that the theory validates all the principles in question, and it is argued that other prima facie plausible principles that the theory fails to validate are objectionable on independent grounds.
\end{abstract}

\section{Implication}

An operation of implication $^{1}$ has traditionally been associated with at least six different (possibly related) functions (which I will refer to as 'the Functions'). Taking an implicational proposition expressed by the conditional $\varphi \rightarrow \psi$, these can informally be expressed as:

SUFFICIENT CONDITION $\Rightarrow$ : Be at least as weak as $\varphi$ being a sufficient condition for $\psi$;

SUFFICIENT CONDITION ${ }^{\Leftarrow}$ : Be at least as strong as $\varphi$ being a sufficient condition for $\psi$;

NO REFUTATION $\Rightarrow$ : Follow from its not being the case that $\varphi$ holds while $\psi$ does not; ${ }^{2}$

NO REFUTATION ${ }^{\leftarrow}$ : Entail that it is not the case that $\varphi$ holds while $\psi$ does not;

DEDUCTION THEOREM: Be at least as weak as $\varphi$ entailing $\psi$;

MODUS PONENS: Together with $\varphi$, logically necessitate $\psi$. $^{3}$

Received September 1, 2012; accepted October 14, 2013

First published online January 28, 2016

2010 Mathematics Subject Classification: Primary 03; Secondary A05, B20, B47, B53, B65

Keywords: analetheism, contraction, dialetheism, naive truth, restricted quantification, substructural logics, transitivity

(C) 2016 by University of Notre Dame $\quad$ 10.1215/00294527-3429057 


\title{
Restriction by Noncontraction
}

\author{
Elia Zardini
}

\begin{abstract}
This paper investigates how naive theories of truth fare with respect to a set of extremely plausible principles of restricted quantification. It is first shown that both nonsubstructural theories as well as certain substructural theories cannot validate all those principles. Then, pursuing further an approach to the semantic paradoxes that the author has defended elsewhere, the theory of restricted quantification available in a specific naive theory that rejects the structural property of contraction is explored. It is shown that the theory validates all the principles in question, and it is argued that other prima facie plausible principles that the theory fails to validate are objectionable on independent grounds.
\end{abstract}

\section{Implication}

An operation of implication $^{1}$ has traditionally been associated with at least six different (possibly related) functions (which I will refer to as 'the Functions'). Taking an implicational proposition expressed by the conditional $\varphi \rightarrow \psi$, these can informally be expressed as:

SUFFICIENT CONDITION $\Rightarrow$ : Be at least as weak as $\varphi$ being a sufficient condition for $\psi$;

SUFFICIENT CONDITION ${ }^{\Leftarrow}$ : Be at least as strong as $\varphi$ being a sufficient condition for $\psi$;

NO REFUTATION $\Rightarrow$ : Follow from its not being the case that $\varphi$ holds while $\psi$ does not; ${ }^{2}$

NO REFUTATION ${ }^{\leftarrow}$ : Entail that it is not the case that $\varphi$ holds while $\psi$ does not;

DEDUCTION THEOREM: Be at least as weak as $\varphi$ entailing $\psi$;

MODUS PONENS: Together with $\varphi$, logically necessitate $\psi$. $^{3}$

Received September 1, 2012; accepted October 14, 2013

First published online January 28, 2016

2010 Mathematics Subject Classification: Primary 03; Secondary A05, B20, B47, B53, B65

Keywords: analetheism, contraction, dialetheism, naive truth, restricted quantification, substructural logics, transitivity

(C) 2016 by University of Notre Dame $\quad$ 10.1215/00294527-3429057 


\title{
Restriction by Noncontraction
}

\author{
Elia Zardini
}

\begin{abstract}
This paper investigates how naive theories of truth fare with respect to a set of extremely plausible principles of restricted quantification. It is first shown that both nonsubstructural theories as well as certain substructural theories cannot validate all those principles. Then, pursuing further an approach to the semantic paradoxes that the author has defended elsewhere, the theory of restricted quantification available in a specific naive theory that rejects the structural property of contraction is explored. It is shown that the theory validates all the principles in question, and it is argued that other prima facie plausible principles that the theory fails to validate are objectionable on independent grounds.
\end{abstract}

\section{Implication}

An operation of implication $^{1}$ has traditionally been associated with at least six different (possibly related) functions (which I will refer to as 'the Functions'). Taking an implicational proposition expressed by the conditional $\varphi \rightarrow \psi$, these can informally be expressed as:

SUFFICIENT CONDITION $\Rightarrow$ : Be at least as weak as $\varphi$ being a sufficient condition for $\psi$;

SUFFICIENT CONDITION ${ }^{\Leftarrow}$ : Be at least as strong as $\varphi$ being a sufficient condition for $\psi$;

NO REFUTATION $\Rightarrow$ : Follow from its not being the case that $\varphi$ holds while $\psi$ does not; ${ }^{2}$

NO REFUTATION ${ }^{\leftarrow}$ : Entail that it is not the case that $\varphi$ holds while $\psi$ does not;

DEDUCTION THEOREM: Be at least as weak as $\varphi$ entailing $\psi$;

MODUS PONENS: Together with $\varphi$, logically necessitate $\psi$. $^{3}$

Received September 1, 2012; accepted October 14, 2013

First published online January 28, 2016

2010 Mathematics Subject Classification: Primary 03; Secondary A05, B20, B47, B53, B65

Keywords: analetheism, contraction, dialetheism, naive truth, restricted quantification, substructural logics, transitivity

(C) 2016 by University of Notre Dame $\quad$ 10.1215/00294527-3429057 


\title{
Restriction by Noncontraction
}

\author{
Elia Zardini
}

\begin{abstract}
This paper investigates how naive theories of truth fare with respect to a set of extremely plausible principles of restricted quantification. It is first shown that both nonsubstructural theories as well as certain substructural theories cannot validate all those principles. Then, pursuing further an approach to the semantic paradoxes that the author has defended elsewhere, the theory of restricted quantification available in a specific naive theory that rejects the structural property of contraction is explored. It is shown that the theory validates all the principles in question, and it is argued that other prima facie plausible principles that the theory fails to validate are objectionable on independent grounds.
\end{abstract}

\section{Implication}

An operation of implication $^{1}$ has traditionally been associated with at least six different (possibly related) functions (which I will refer to as 'the Functions'). Taking an implicational proposition expressed by the conditional $\varphi \rightarrow \psi$, these can informally be expressed as:

SUFFICIENT CONDITION $\Rightarrow$ : Be at least as weak as $\varphi$ being a sufficient condition for $\psi$;

SUFFICIENT CONDITION ${ }^{\Leftarrow}$ : Be at least as strong as $\varphi$ being a sufficient condition for $\psi$;

NO REFUTATION $\Rightarrow$ : Follow from its not being the case that $\varphi$ holds while $\psi$ does not; ${ }^{2}$

NO REFUTATION ${ }^{\leftarrow}$ : Entail that it is not the case that $\varphi$ holds while $\psi$ does not;

DEDUCTION THEOREM: Be at least as weak as $\varphi$ entailing $\psi$;

MODUS PONENS: Together with $\varphi$, logically necessitate $\psi$. $^{3}$

Received September 1, 2012; accepted October 14, 2013

First published online January 28, 2016

2010 Mathematics Subject Classification: Primary 03; Secondary A05, B20, B47, B53, B65

Keywords: analetheism, contraction, dialetheism, naive truth, restricted quantification, substructural logics, transitivity

(C) 2016 by University of Notre Dame $\quad$ 10.1215/00294527-3429057 


\title{
Restriction by Noncontraction
}

\author{
Elia Zardini
}

\begin{abstract}
This paper investigates how naive theories of truth fare with respect to a set of extremely plausible principles of restricted quantification. It is first shown that both nonsubstructural theories as well as certain substructural theories cannot validate all those principles. Then, pursuing further an approach to the semantic paradoxes that the author has defended elsewhere, the theory of restricted quantification available in a specific naive theory that rejects the structural property of contraction is explored. It is shown that the theory validates all the principles in question, and it is argued that other prima facie plausible principles that the theory fails to validate are objectionable on independent grounds.
\end{abstract}

\section{Implication}

An operation of implication $^{1}$ has traditionally been associated with at least six different (possibly related) functions (which I will refer to as 'the Functions'). Taking an implicational proposition expressed by the conditional $\varphi \rightarrow \psi$, these can informally be expressed as:

SUFFICIENT CONDITION $\Rightarrow$ : Be at least as weak as $\varphi$ being a sufficient condition for $\psi$;

SUFFICIENT CONDITION ${ }^{\Leftarrow}$ : Be at least as strong as $\varphi$ being a sufficient condition for $\psi$;

NO REFUTATION $\Rightarrow$ : Follow from its not being the case that $\varphi$ holds while $\psi$ does not; ${ }^{2}$

NO REFUTATION ${ }^{\leftarrow}$ : Entail that it is not the case that $\varphi$ holds while $\psi$ does not;

DEDUCTION THEOREM: Be at least as weak as $\varphi$ entailing $\psi$;

MODUS PONENS: Together with $\varphi$, logically necessitate $\psi$. $^{3}$

Received September 1, 2012; accepted October 14, 2013

First published online January 28, 2016

2010 Mathematics Subject Classification: Primary 03; Secondary A05, B20, B47, B53, B65

Keywords: analetheism, contraction, dialetheism, naive truth, restricted quantification, substructural logics, transitivity

(C) 2016 by University of Notre Dame $\quad$ 10.1215/00294527-3429057 


\title{
Restriction by Noncontraction
}

\author{
Elia Zardini
}

\begin{abstract}
This paper investigates how naive theories of truth fare with respect to a set of extremely plausible principles of restricted quantification. It is first shown that both nonsubstructural theories as well as certain substructural theories cannot validate all those principles. Then, pursuing further an approach to the semantic paradoxes that the author has defended elsewhere, the theory of restricted quantification available in a specific naive theory that rejects the structural property of contraction is explored. It is shown that the theory validates all the principles in question, and it is argued that other prima facie plausible principles that the theory fails to validate are objectionable on independent grounds.
\end{abstract}

\section{Implication}

An operation of implication $^{1}$ has traditionally been associated with at least six different (possibly related) functions (which I will refer to as 'the Functions'). Taking an implicational proposition expressed by the conditional $\varphi \rightarrow \psi$, these can informally be expressed as:

SUFFICIENT CONDITION $\Rightarrow$ : Be at least as weak as $\varphi$ being a sufficient condition for $\psi$;

SUFFICIENT CONDITION ${ }^{\Leftarrow}$ : Be at least as strong as $\varphi$ being a sufficient condition for $\psi$;

NO REFUTATION $\Rightarrow$ : Follow from its not being the case that $\varphi$ holds while $\psi$ does not; ${ }^{2}$

NO REFUTATION ${ }^{\leftarrow}$ : Entail that it is not the case that $\varphi$ holds while $\psi$ does not;

DEDUCTION THEOREM: Be at least as weak as $\varphi$ entailing $\psi$;

MODUS PONENS: Together with $\varphi$, logically necessitate $\psi$. $^{3}$

Received September 1, 2012; accepted October 14, 2013

First published online January 28, 2016

2010 Mathematics Subject Classification: Primary 03; Secondary A05, B20, B47, B53, B65

Keywords: analetheism, contraction, dialetheism, naive truth, restricted quantification, substructural logics, transitivity

(C) 2016 by University of Notre Dame $\quad$ 10.1215/00294527-3429057 


\title{
Restriction by Noncontraction
}

\author{
Elia Zardini
}

\begin{abstract}
This paper investigates how naive theories of truth fare with respect to a set of extremely plausible principles of restricted quantification. It is first shown that both nonsubstructural theories as well as certain substructural theories cannot validate all those principles. Then, pursuing further an approach to the semantic paradoxes that the author has defended elsewhere, the theory of restricted quantification available in a specific naive theory that rejects the structural property of contraction is explored. It is shown that the theory validates all the principles in question, and it is argued that other prima facie plausible principles that the theory fails to validate are objectionable on independent grounds.
\end{abstract}

\section{Implication}

An operation of implication $^{1}$ has traditionally been associated with at least six different (possibly related) functions (which I will refer to as 'the Functions'). Taking an implicational proposition expressed by the conditional $\varphi \rightarrow \psi$, these can informally be expressed as:

SUFFICIENT CONDITION $\Rightarrow$ : Be at least as weak as $\varphi$ being a sufficient condition for $\psi$;

SUFFICIENT CONDITION ${ }^{\Leftarrow}$ : Be at least as strong as $\varphi$ being a sufficient condition for $\psi$;

NO REFUTATION $\Rightarrow$ : Follow from its not being the case that $\varphi$ holds while $\psi$ does not; ${ }^{2}$

NO REFUTATION ${ }^{\leftarrow}$ : Entail that it is not the case that $\varphi$ holds while $\psi$ does not;

DEDUCTION THEOREM: Be at least as weak as $\varphi$ entailing $\psi$;

MODUS PONENS: Together with $\varphi$, logically necessitate $\psi$. $^{3}$

Received September 1, 2012; accepted October 14, 2013

First published online January 28, 2016

2010 Mathematics Subject Classification: Primary 03; Secondary A05, B20, B47, B53, B65

Keywords: analetheism, contraction, dialetheism, naive truth, restricted quantification, substructural logics, transitivity

(C) 2016 by University of Notre Dame $\quad$ 10.1215/00294527-3429057 


\title{
Restriction by Noncontraction
}

\author{
Elia Zardini
}

\begin{abstract}
This paper investigates how naive theories of truth fare with respect to a set of extremely plausible principles of restricted quantification. It is first shown that both nonsubstructural theories as well as certain substructural theories cannot validate all those principles. Then, pursuing further an approach to the semantic paradoxes that the author has defended elsewhere, the theory of restricted quantification available in a specific naive theory that rejects the structural property of contraction is explored. It is shown that the theory validates all the principles in question, and it is argued that other prima facie plausible principles that the theory fails to validate are objectionable on independent grounds.
\end{abstract}

\section{Implication}

An operation of implication $^{1}$ has traditionally been associated with at least six different (possibly related) functions (which I will refer to as 'the Functions'). Taking an implicational proposition expressed by the conditional $\varphi \rightarrow \psi$, these can informally be expressed as:

SUFFICIENT CONDITION $\Rightarrow$ : Be at least as weak as $\varphi$ being a sufficient condition for $\psi$;

SUFFICIENT CONDITION ${ }^{\Leftarrow}$ : Be at least as strong as $\varphi$ being a sufficient condition for $\psi$;

NO REFUTATION $\Rightarrow$ : Follow from its not being the case that $\varphi$ holds while $\psi$ does not; ${ }^{2}$

NO REFUTATION ${ }^{\leftarrow}$ : Entail that it is not the case that $\varphi$ holds while $\psi$ does not;

DEDUCTION THEOREM: Be at least as weak as $\varphi$ entailing $\psi$;

MODUS PONENS: Together with $\varphi$, logically necessitate $\psi$. $^{3}$

Received September 1, 2012; accepted October 14, 2013

First published online January 28, 2016

2010 Mathematics Subject Classification: Primary 03; Secondary A05, B20, B47, B53, B65

Keywords: analetheism, contraction, dialetheism, naive truth, restricted quantification, substructural logics, transitivity

(C) 2016 by University of Notre Dame $\quad$ 10.1215/00294527-3429057 


\title{
Restriction by Noncontraction
}

\author{
Elia Zardini
}

\begin{abstract}
This paper investigates how naive theories of truth fare with respect to a set of extremely plausible principles of restricted quantification. It is first shown that both nonsubstructural theories as well as certain substructural theories cannot validate all those principles. Then, pursuing further an approach to the semantic paradoxes that the author has defended elsewhere, the theory of restricted quantification available in a specific naive theory that rejects the structural property of contraction is explored. It is shown that the theory validates all the principles in question, and it is argued that other prima facie plausible principles that the theory fails to validate are objectionable on independent grounds.
\end{abstract}

\section{Implication}

An operation of implication $^{1}$ has traditionally been associated with at least six different (possibly related) functions (which I will refer to as 'the Functions'). Taking an implicational proposition expressed by the conditional $\varphi \rightarrow \psi$, these can informally be expressed as:

SUFFICIENT CONDITION $\Rightarrow$ : Be at least as weak as $\varphi$ being a sufficient condition for $\psi$;

SUFFICIENT CONDITION ${ }^{\Leftarrow}$ : Be at least as strong as $\varphi$ being a sufficient condition for $\psi$;

NO REFUTATION $\Rightarrow$ : Follow from its not being the case that $\varphi$ holds while $\psi$ does not; ${ }^{2}$

NO REFUTATION ${ }^{\leftarrow}$ : Entail that it is not the case that $\varphi$ holds while $\psi$ does not;

DEDUCTION THEOREM: Be at least as weak as $\varphi$ entailing $\psi$;

MODUS PONENS: Together with $\varphi$, logically necessitate $\psi$. $^{3}$

Received September 1, 2012; accepted October 14, 2013

First published online January 28, 2016

2010 Mathematics Subject Classification: Primary 03; Secondary A05, B20, B47, B53, B65

Keywords: analetheism, contraction, dialetheism, naive truth, restricted quantification, substructural logics, transitivity

(C) 2016 by University of Notre Dame $\quad$ 10.1215/00294527-3429057 


\title{
Restriction by Noncontraction
}

\author{
Elia Zardini
}

\begin{abstract}
This paper investigates how naive theories of truth fare with respect to a set of extremely plausible principles of restricted quantification. It is first shown that both nonsubstructural theories as well as certain substructural theories cannot validate all those principles. Then, pursuing further an approach to the semantic paradoxes that the author has defended elsewhere, the theory of restricted quantification available in a specific naive theory that rejects the structural property of contraction is explored. It is shown that the theory validates all the principles in question, and it is argued that other prima facie plausible principles that the theory fails to validate are objectionable on independent grounds.
\end{abstract}

\section{Implication}

An operation of implication $^{1}$ has traditionally been associated with at least six different (possibly related) functions (which I will refer to as 'the Functions'). Taking an implicational proposition expressed by the conditional $\varphi \rightarrow \psi$, these can informally be expressed as:

SUFFICIENT CONDITION $\Rightarrow$ : Be at least as weak as $\varphi$ being a sufficient condition for $\psi$;

SUFFICIENT CONDITION ${ }^{\Leftarrow}$ : Be at least as strong as $\varphi$ being a sufficient condition for $\psi$;

NO REFUTATION $\Rightarrow$ : Follow from its not being the case that $\varphi$ holds while $\psi$ does not; ${ }^{2}$

NO REFUTATION ${ }^{\leftarrow}$ : Entail that it is not the case that $\varphi$ holds while $\psi$ does not;

DEDUCTION THEOREM: Be at least as weak as $\varphi$ entailing $\psi$;

MODUS PONENS: Together with $\varphi$, logically necessitate $\psi$. $^{3}$

Received September 1, 2012; accepted October 14, 2013

First published online January 28, 2016

2010 Mathematics Subject Classification: Primary 03; Secondary A05, B20, B47, B53, B65

Keywords: analetheism, contraction, dialetheism, naive truth, restricted quantification, substructural logics, transitivity

(C) 2016 by University of Notre Dame $\quad$ 10.1215/00294527-3429057 


\title{
Restriction by Noncontraction
}

\author{
Elia Zardini
}

\begin{abstract}
This paper investigates how naive theories of truth fare with respect to a set of extremely plausible principles of restricted quantification. It is first shown that both nonsubstructural theories as well as certain substructural theories cannot validate all those principles. Then, pursuing further an approach to the semantic paradoxes that the author has defended elsewhere, the theory of restricted quantification available in a specific naive theory that rejects the structural property of contraction is explored. It is shown that the theory validates all the principles in question, and it is argued that other prima facie plausible principles that the theory fails to validate are objectionable on independent grounds.
\end{abstract}

\section{Implication}

An operation of implication $^{1}$ has traditionally been associated with at least six different (possibly related) functions (which I will refer to as 'the Functions'). Taking an implicational proposition expressed by the conditional $\varphi \rightarrow \psi$, these can informally be expressed as:

SUFFICIENT CONDITION $\Rightarrow$ : Be at least as weak as $\varphi$ being a sufficient condition for $\psi$;

SUFFICIENT CONDITION ${ }^{\Leftarrow}$ : Be at least as strong as $\varphi$ being a sufficient condition for $\psi$;

NO REFUTATION $\Rightarrow$ : Follow from its not being the case that $\varphi$ holds while $\psi$ does not; ${ }^{2}$

NO REFUTATION ${ }^{\leftarrow}$ : Entail that it is not the case that $\varphi$ holds while $\psi$ does not;

DEDUCTION THEOREM: Be at least as weak as $\varphi$ entailing $\psi$;

MODUS PONENS: Together with $\varphi$, logically necessitate $\psi$. $^{3}$

Received September 1, 2012; accepted October 14, 2013

First published online January 28, 2016

2010 Mathematics Subject Classification: Primary 03; Secondary A05, B20, B47, B53, B65

Keywords: analetheism, contraction, dialetheism, naive truth, restricted quantification, substructural logics, transitivity

(C) 2016 by University of Notre Dame $\quad$ 10.1215/00294527-3429057 


\title{
Restriction by Noncontraction
}

\author{
Elia Zardini
}

\begin{abstract}
This paper investigates how naive theories of truth fare with respect to a set of extremely plausible principles of restricted quantification. It is first shown that both nonsubstructural theories as well as certain substructural theories cannot validate all those principles. Then, pursuing further an approach to the semantic paradoxes that the author has defended elsewhere, the theory of restricted quantification available in a specific naive theory that rejects the structural property of contraction is explored. It is shown that the theory validates all the principles in question, and it is argued that other prima facie plausible principles that the theory fails to validate are objectionable on independent grounds.
\end{abstract}

\section{Implication}

An operation of implication $^{1}$ has traditionally been associated with at least six different (possibly related) functions (which I will refer to as 'the Functions'). Taking an implicational proposition expressed by the conditional $\varphi \rightarrow \psi$, these can informally be expressed as:

SUFFICIENT CONDITION $\Rightarrow$ : Be at least as weak as $\varphi$ being a sufficient condition for $\psi$;

SUFFICIENT CONDITION ${ }^{\Leftarrow}$ : Be at least as strong as $\varphi$ being a sufficient condition for $\psi$;

NO REFUTATION $\Rightarrow$ : Follow from its not being the case that $\varphi$ holds while $\psi$ does not; ${ }^{2}$

NO REFUTATION ${ }^{\leftarrow}$ : Entail that it is not the case that $\varphi$ holds while $\psi$ does not;

DEDUCTION THEOREM: Be at least as weak as $\varphi$ entailing $\psi$;

MODUS PONENS: Together with $\varphi$, logically necessitate $\psi$. $^{3}$

Received September 1, 2012; accepted October 14, 2013

First published online January 28, 2016

2010 Mathematics Subject Classification: Primary 03; Secondary A05, B20, B47, B53, B65

Keywords: analetheism, contraction, dialetheism, naive truth, restricted quantification, substructural logics, transitivity

(C) 2016 by University of Notre Dame $\quad$ 10.1215/00294527-3429057 


\title{
Restriction by Noncontraction
}

\author{
Elia Zardini
}

\begin{abstract}
This paper investigates how naive theories of truth fare with respect to a set of extremely plausible principles of restricted quantification. It is first shown that both nonsubstructural theories as well as certain substructural theories cannot validate all those principles. Then, pursuing further an approach to the semantic paradoxes that the author has defended elsewhere, the theory of restricted quantification available in a specific naive theory that rejects the structural property of contraction is explored. It is shown that the theory validates all the principles in question, and it is argued that other prima facie plausible principles that the theory fails to validate are objectionable on independent grounds.
\end{abstract}

\section{Implication}

An operation of implication $^{1}$ has traditionally been associated with at least six different (possibly related) functions (which I will refer to as 'the Functions'). Taking an implicational proposition expressed by the conditional $\varphi \rightarrow \psi$, these can informally be expressed as:

SUFFICIENT CONDITION $\Rightarrow$ : Be at least as weak as $\varphi$ being a sufficient condition for $\psi$;

SUFFICIENT CONDITION ${ }^{\Leftarrow}$ : Be at least as strong as $\varphi$ being a sufficient condition for $\psi$;

NO REFUTATION $\Rightarrow$ : Follow from its not being the case that $\varphi$ holds while $\psi$ does not; ${ }^{2}$

NO REFUTATION ${ }^{\leftarrow}$ : Entail that it is not the case that $\varphi$ holds while $\psi$ does not;

DEDUCTION THEOREM: Be at least as weak as $\varphi$ entailing $\psi$;

MODUS PONENS: Together with $\varphi$, logically necessitate $\psi$. $^{3}$

Received September 1, 2012; accepted October 14, 2013

First published online January 28, 2016

2010 Mathematics Subject Classification: Primary 03; Secondary A05, B20, B47, B53, B65

Keywords: analetheism, contraction, dialetheism, naive truth, restricted quantification, substructural logics, transitivity

(C) 2016 by University of Notre Dame $\quad$ 10.1215/00294527-3429057 


\title{
Restriction by Noncontraction
}

\author{
Elia Zardini
}

\begin{abstract}
This paper investigates how naive theories of truth fare with respect to a set of extremely plausible principles of restricted quantification. It is first shown that both nonsubstructural theories as well as certain substructural theories cannot validate all those principles. Then, pursuing further an approach to the semantic paradoxes that the author has defended elsewhere, the theory of restricted quantification available in a specific naive theory that rejects the structural property of contraction is explored. It is shown that the theory validates all the principles in question, and it is argued that other prima facie plausible principles that the theory fails to validate are objectionable on independent grounds.
\end{abstract}

\section{Implication}

An operation of implication $^{1}$ has traditionally been associated with at least six different (possibly related) functions (which I will refer to as 'the Functions'). Taking an implicational proposition expressed by the conditional $\varphi \rightarrow \psi$, these can informally be expressed as:

SUFFICIENT CONDITION $\Rightarrow$ : Be at least as weak as $\varphi$ being a sufficient condition for $\psi$;

SUFFICIENT CONDITION ${ }^{\Leftarrow}$ : Be at least as strong as $\varphi$ being a sufficient condition for $\psi$;

NO REFUTATION $\Rightarrow$ : Follow from its not being the case that $\varphi$ holds while $\psi$ does not; ${ }^{2}$

NO REFUTATION ${ }^{\leftarrow}$ : Entail that it is not the case that $\varphi$ holds while $\psi$ does not;

DEDUCTION THEOREM: Be at least as weak as $\varphi$ entailing $\psi$;

MODUS PONENS: Together with $\varphi$, logically necessitate $\psi$. $^{3}$

Received September 1, 2012; accepted October 14, 2013

First published online January 28, 2016

2010 Mathematics Subject Classification: Primary 03; Secondary A05, B20, B47, B53, B65

Keywords: analetheism, contraction, dialetheism, naive truth, restricted quantification, substructural logics, transitivity

(C) 2016 by University of Notre Dame $\quad$ 10.1215/00294527-3429057 


\title{
Restriction by Noncontraction
}

\author{
Elia Zardini
}

\begin{abstract}
This paper investigates how naive theories of truth fare with respect to a set of extremely plausible principles of restricted quantification. It is first shown that both nonsubstructural theories as well as certain substructural theories cannot validate all those principles. Then, pursuing further an approach to the semantic paradoxes that the author has defended elsewhere, the theory of restricted quantification available in a specific naive theory that rejects the structural property of contraction is explored. It is shown that the theory validates all the principles in question, and it is argued that other prima facie plausible principles that the theory fails to validate are objectionable on independent grounds.
\end{abstract}

\section{Implication}

An operation of implication $^{1}$ has traditionally been associated with at least six different (possibly related) functions (which I will refer to as 'the Functions'). Taking an implicational proposition expressed by the conditional $\varphi \rightarrow \psi$, these can informally be expressed as:

SUFFICIENT CONDITION $\Rightarrow$ : Be at least as weak as $\varphi$ being a sufficient condition for $\psi$;

SUFFICIENT CONDITION ${ }^{\Leftarrow}$ : Be at least as strong as $\varphi$ being a sufficient condition for $\psi$;

NO REFUTATION $\Rightarrow$ : Follow from its not being the case that $\varphi$ holds while $\psi$ does not; ${ }^{2}$

NO REFUTATION ${ }^{\leftarrow}$ : Entail that it is not the case that $\varphi$ holds while $\psi$ does not;

DEDUCTION THEOREM: Be at least as weak as $\varphi$ entailing $\psi$;

MODUS PONENS: Together with $\varphi$, logically necessitate $\psi$. $^{3}$

Received September 1, 2012; accepted October 14, 2013

First published online January 28, 2016

2010 Mathematics Subject Classification: Primary 03; Secondary A05, B20, B47, B53, B65

Keywords: analetheism, contraction, dialetheism, naive truth, restricted quantification, substructural logics, transitivity

(C) 2016 by University of Notre Dame $\quad$ 10.1215/00294527-3429057 


\title{
Restriction by Noncontraction
}

\author{
Elia Zardini
}

\begin{abstract}
This paper investigates how naive theories of truth fare with respect to a set of extremely plausible principles of restricted quantification. It is first shown that both nonsubstructural theories as well as certain substructural theories cannot validate all those principles. Then, pursuing further an approach to the semantic paradoxes that the author has defended elsewhere, the theory of restricted quantification available in a specific naive theory that rejects the structural property of contraction is explored. It is shown that the theory validates all the principles in question, and it is argued that other prima facie plausible principles that the theory fails to validate are objectionable on independent grounds.
\end{abstract}

\section{Implication}

An operation of implication $^{1}$ has traditionally been associated with at least six different (possibly related) functions (which I will refer to as 'the Functions'). Taking an implicational proposition expressed by the conditional $\varphi \rightarrow \psi$, these can informally be expressed as:

SUFFICIENT CONDITION $\Rightarrow$ : Be at least as weak as $\varphi$ being a sufficient condition for $\psi$;

SUFFICIENT CONDITION ${ }^{\Leftarrow}$ : Be at least as strong as $\varphi$ being a sufficient condition for $\psi$;

NO REFUTATION $\Rightarrow$ : Follow from its not being the case that $\varphi$ holds while $\psi$ does not; ${ }^{2}$

NO REFUTATION ${ }^{\leftarrow}$ : Entail that it is not the case that $\varphi$ holds while $\psi$ does not;

DEDUCTION THEOREM: Be at least as weak as $\varphi$ entailing $\psi$;

MODUS PONENS: Together with $\varphi$, logically necessitate $\psi$. $^{3}$

Received September 1, 2012; accepted October 14, 2013

First published online January 28, 2016

2010 Mathematics Subject Classification: Primary 03; Secondary A05, B20, B47, B53, B65

Keywords: analetheism, contraction, dialetheism, naive truth, restricted quantification, substructural logics, transitivity

(C) 2016 by University of Notre Dame $\quad$ 10.1215/00294527-3429057 